\title{
CONSTRUCTION OF THE HALF-LINE POTENTIAL FROM THE JOST FUNCTION
}

\author{
Tuncay Aktosun \\ Department of Mathematics and Statistics \\ Mississippi State University \\ Mississippi State, MS 39762
}

\begin{abstract}
For the one-dimensional Schrödinger equation, the analysis is provided to recover the portion of the potential lying to the right (left) of any chosen point. The scattering data used consists of the left (right) Jost solution or its spatial derivative evaluated at that point, or the amplitudes of such functions. Various uniqueness and nonuniqueness results are established, and the recovery is illustrated with some explicit examples.
\end{abstract}

PACS (2003): 02.30.Zz, 03.65.Nk

Mathematics Subject Classification (2000): 34A55, 34L25, 34L40, 47A40, 81U05

Keywords: Schrödinger equation, Inverse scattering, Jost solution, Recovery of potential Short title: Construction of the half-line potential 


\section{INTRODUCTION}

In this paper we consider the one-dimensional Schrödinger equation and analyze the recovery of the portion of the potential lying to the right (left) of any chosen point. As our scattering data we use the amplitude of the Jost solution from the left (right) evaluated at that chosen point, or we use the amplitude of the spatial derivative of that Jost solution. As the alternate scattering data, we also consider using the Jost solution evaluated at the chosen point rather than its amplitude, or the spatial derivative of that Jost solution rather than the amplitude of that spatial derivative.

Without loss of any generality, our problem can be reduced to the recovery of the potential with support in $\mathbf{R}^{+}$in terms of $\left|f_{1}(k, 0)\right|$, or $\left|f_{1}^{\prime}(k, 0)\right|, f_{1}(k, 0)$, or $f_{1}^{\prime}(k, 0)$, where $f_{1}(k, x)$ denotes the left Jost solution to the Schrödinger equation

$$
\Psi^{\prime \prime}(k, x)+k^{2} \Psi(k, x)=V(x) \Psi(k, x), \quad x \in \mathbf{R},
$$

and the prime denotes the derivative with respect to the spatial coordinate $x$. Throughout our paper we assume that the potential $V$ belongs to the class $\mathcal{W}$; that is, $V(x)=c_{0} \delta(x)+$ $U(x)$, where $c_{0}$ is a real constant, $\delta(x)$ is the Dirac delta distribution, and $U$ is a real-valued potential having no bound states, vanishing for $x<0$, and belonging to $L_{1}^{1}\left(\mathbf{R}^{+}\right)$. By $L_{n}^{1}(J)$ we denote the class of Lebesgue-measurable potentials $U$ on an interval $J$ such that the integral $\int_{J} d x\left(1+|x|^{n}\right)|U(x)|$ is finite. For a review of the direct and inverse scattering problems for the Schrödinger equation on the line, the reader is referred to [1-6].

The following question was raised by Roy Pike while the author was attending the RCP264 Workshop on Inverse Problems and Nonlinearity in Montpellier, France in June 2000: In the absence of bound states, does $f_{1}^{\prime}(k, 0)$ uniquely determine $U$ ? While the author was attending the IMA Summer Program on Geometric Methods in Inverse Problems in Minneapolis in July 2001, a solution to this problem was suggested by John Sylvester based on the observation that the real part of $[1+L(k)] /[1-L(k)]$ can be expressed as 
$k^{2} /\left|f_{1}^{\prime}(k, 0)\right|^{2}$, where $L$ is the reflection coefficient from the left corresponding to (1.1). Let us use $\mathbf{C}^{+}$to denote the upper half complex plane and let $\overline{\mathbf{C}^{+}}:=\mathbf{C}^{+} \cup \mathbf{R}$. Note that a function that is analytic in $\mathbf{C}^{+}$and continuous and bounded in $\overline{\mathbf{C}^{+}}$can be constructed uniquely if the real part of that function is known on the real axis $\mathbf{R}$. Thus, by using the Schwarz integral formula (the Poisson integral formula for the half plane) [7], one can construct $L$ from the data containing the real part of $L$. Having constructed $L$, the potential is then uniquely obtained from $L$ via one of the known methods [1-6]. It is already known that $L$ uniquely determines $V$ even when $V$ has bound states [8-13] as a consequence of $V(x) \equiv 0$ for $x<0$; no extra information on bound states is needed to recover $V$ from $L$; in fact, the information on bound-state energies and norming constants is already hidden in $L(k)$ as the poles and residues at those poles, respectively, [cf. Theorem 2.1 (iv)] of the meromorphic extension of $L(k)$ in $\mathbf{C}^{+}$.

Recall that the left Jost solution $f_{1}(k, x)$ to $(1.1)$ satisfies the boundary conditions

$$
f_{1}(k, x)=e^{i k x}[1+o(1)], \quad f_{1}^{\prime}(k, x)=i k e^{i k x}[1+o(1)], \quad x \rightarrow+\infty .
$$

Since the potential $V$ is assumed to vanish on $\mathbf{R}^{-}$, the transmission coefficient $T$ and the left reflection coefficient $L$ are related to $f_{1}(k, 0)$ and $f_{1}^{\prime}\left(k, 0^{-}\right)$as

$$
f_{1}(k, 0)=\frac{1+L(k)}{T(k)}, \quad f_{1}^{\prime}\left(k, 0^{-}\right)=i k \frac{1-L(k)}{T(k)} .
$$

Due to a possible Dirac delta distribution of strength $c_{0}$ at $x=0, f_{1}^{\prime}(k, x)$ may have a jump discontinuity at $x=0$, whereas $f_{1}(k, x)$ is unaffected by $c_{0}$ and is continuous in $x$ at $x=0$. We have

$$
f_{1}\left(k, 0^{+}\right)=f_{1}\left(k, 0^{-}\right), \quad f_{1}^{\prime}\left(k, 0^{-}\right)=f_{1}^{\prime}\left(k, 0^{+}\right)-c_{0} f_{1}(k, 0) .
$$

The presence of $c_{0}$ somehow complicates the problem we study. For example, even though $U$ is assumed to have no bound states, the potential $V$ has a bound state if $c_{0}$ is negative enough, as indicated in Proposition 2.1. 
Let us use an asterisk to denote complex conjugation. Using [1-6]

$$
f_{\mathrm{l}}(-k, x)=f_{\mathrm{l}}(k, x)^{*}, \quad f_{\mathrm{l}}^{\prime}(-k, x)=f_{\mathrm{l}}^{\prime}(k, x)^{*}, \quad x \in \mathbf{R},
$$

in our scattering data it is sufficient to supply $\left|f_{1}(k, 0)\right|$ or $\left|f_{1}^{\prime}\left(k, 0^{-}\right)\right|$only for $k \in \mathbf{R}^{+}$. Alternatively, as the scattering data one can use $f_{1}(k, 0)$ or $f_{1}^{\prime}\left(k, 0^{-}\right)$given on a subinterval of the real axis as a result of the uniqueness of the meromorphic continuation from $\mathbf{R}$ to $\mathbf{C}^{+}$.

Recall that for the radial Schrödinger equation, $f_{1}(k, 0)$ is usually known as the Jost function. Even though this terminology is in general not used for the full-line problem, we are using it in the title of our article. Our paper is organized as follows. In Section 2 we present the relevant properties of $f_{1}(k, 0)$ and $f_{1}^{\prime}(k, 0)$ corresponding to a potential belonging to class $\mathcal{W}$; we also indicate the characterization for $L$ corresponding to a potential in $\mathcal{W}$. In Section 3 we show that, as far as constructing potentials in $\mathcal{W}$ is concerned, knowledge of $\left|f_{1}(k, 0)\right|$ is equivalent to knowledge of $f_{1}(k, 0)$, but knowledge of $\left|f_{1}^{\prime}(k, 0)\right|$ is equivalent to knowledge of $f_{1}^{\prime}(k, 0)$ only when there are no bound states. In Section 4 we present the construction of $L$ in terms of the data $f_{1}(k, 0)$ or equivalently $\left|f_{1}(k, 0)\right|$. Finally, in Section 5 we present the construction of $L$ in terms of the data $f_{1}^{\prime}(k, 0)$ or $\left|f_{1}^{\prime}(k, 0)\right|$. Our findings are summarized in the following theorem:

Theorem 1.1 Assume $V$ belongs to class $\mathcal{W}$ and let $f_{1}(k, x)$ denote the corresponding left Jost solution. We have the following:

(i) There exists a one-parameter family of potentials in $\mathcal{W}$ corresponding to the scattering data $\left\{f_{1}(k, 0): k \in \mathbf{R}\right\}$ or equivalently corresponding to $\left\{\left|f_{1}(k, 0)\right|: k \in \mathbf{R}\right\}$. The parameter can be chosen as $c_{0}$, the strength of the Dirac delta distribution in the potential. Hence, if the scattering data contains the value of $c_{0}$, it corresponds to a unique potential in $\mathcal{W}$.

(ii) If $V$ does not contain a bound state, then the correspondence between $V$ and the scattering data $\left\{f_{1}^{\prime}(k, 0): k \in \mathbf{R}\right\}$ is one-to-one. If $V$ contains a bound state, then 
there exists a one-parameter family of potentials in $\mathcal{W}$ corresponding to the scattering data $\left\{f_{1}^{\prime}(k, 0): k \in \mathbf{R}\right\}$; the correspondence can be made one-to-one by also specifying the value of $f_{1}(0,0)$ in the scattering data or equivalently by specifying the value of $c_{0}$.

(iii) If $V$ contains a bound state, then there exists a two-parameter family of potentials in $\mathcal{W}$ corresponding to the scattering data $\left\{\left|f_{1}^{\prime}(k, 0)\right|: k \in \mathbf{R}\right\}$. The correspondence can be made one-to-one by also specifying any two of the three quantities $f_{1}(0,0), c_{0}$, and the bound-state energy in the scattering data. Alternatively, in order to have a one-toone correspondence, one can specify also any two of the three quantities $f_{1}(0,0)$, the strength of the Dirac delta distribution in the potential, and the only zero of $\left|f_{1}^{\prime}(k, 0)\right|$ in $\mathbf{C}^{+}$in the scattering data.

Returning to Pike's question, we obtain the following conclusion from the above theorem: A potential with support in $\mathbf{R}^{+}$having no bound states and no Dirac delta distributions is uniquely determined from either $f_{1}^{\prime}(k, 0)$ or $\left|f_{1}^{\prime}(k, 0)\right|$.

As noted by Ricardo Weder, when $c_{0} \equiv 0$, the result of (i) in the above theorem already follows from the Gel'fand-Levitan theory $[4,5,14]$ for the radial Schrödinger equation, where $\left|f_{1}(k, 0)\right|$ is used as the input to the spectral function; thus, the existence, uniqueness, and reconstruction of $U$ from $f_{1}(k, 0)$ or $\left|f_{1}(k, 0)\right|$ are also obtained via the solution of the radial Gel'fand-Levitan integral equation. Similarly, when $c_{0} \equiv 0$, the data $f_{1}(k, 0)$ uniquely constructs the radial scattering matrix $S(k):=\frac{f_{1}(-k, 0)}{f_{1}(k, 0)}$, which is used as the input to the Marchenko integral equation $[4,5,14,15]$ for the radial Schrödinger equation; thus, the existence, uniqueness, and reconstruction of $U$ from $f_{1}(k, 0)$ are also obtained via the solution of the radial Marchenko integral equation. In that regard, our results in this paper using the data $\left|f_{1}(k, 0)\right|$ or $f_{1}(k, 0)$ can be viewed as also the analysis of the existence, uniqueness, and reconstruction of $f_{1}^{\prime}\left(k, 0^{-}\right)$from the available data. Similarly, our results using the data $\left|f_{1}^{\prime}\left(k, 0^{-}\right)\right|$or $f_{1}^{\prime}\left(k, 0^{-}\right)$can also be interpreted as the analysis of the existence, uniqueness, and reconstruction of $f_{1}(k, 0)$ from the available data. 


\section{PRELIMINARIES}

For the Schrödinger equation one needs to make a distinction between the generic case and the exceptional case. Recall that generically $T(0)=0$ and in the exceptional case $T(0) \neq 0$. One can also characterize the generic case as $f_{1}^{\prime}\left(0,0^{-}\right) \neq 0$ and the exceptional case as $f_{1}^{\prime}\left(0,0^{-}\right)=0$; alternatively, we have $L(0)=-1$ generically and $L(0) \in(-1,1)$ in the exceptional case. An exceptional case signals the borderline case of adding or subtracting a bound state. Within the class $\mathcal{W}$, since $U$ has no bound states, $V$ has either zero or one bound state [16]. The following proposition gives the exact criterion on the number of bound states of $V$.

Proposition 2.1 Let $V$ belong to class $\mathcal{W}, c_{0}$ denote the strength of the Dirac delta distribution in $V$ at $x=0$, and $U(x):=V(x)-c_{0} \delta(x)$. Then, there exists a nonpositive constant $\tilde{c}_{0}$, uniquely determined by $U$ alone as in (2.1), and we have:

(i) If $c_{0}>\tilde{c}_{0}$, then $V$ is generic and has no bound states. In this case, $f_{1}(0,0)>0$, $f_{1}^{\prime}\left(0,0^{-}\right)<0$, and $T(0)=0$; moreover, for $z>0$ we have $f_{1}(i z, 0)>0, f_{1}^{\prime}\left(i z, 0^{-}\right)<0$, and $T(i z)>0$.

(ii) If $c_{0}=\tilde{c}_{0}$, then $V$ is exceptional and has no bound states. In this case, $f_{1}(0,0)>0$, $f_{1}^{\prime}\left(0,0^{-}\right)=0$, and $T(0)>0$; moreover, for $z>0$ we have $f_{1}(i z, 0)>0, f_{1}^{\prime}\left(i z, 0^{-}\right)<0$, and $T(i z)>0$.

(iii) If $c_{0}<\tilde{c}_{0}$, then $V$ is generic and has exactly one bound state at $k=i \kappa$ for some $\kappa>0$, i.e. $1 / T(i \kappa)=0$. In this case, $f_{1}(0,0)>0, f_{1}^{\prime}\left(0,0^{-}\right)>0, T(0)=0$, and there exists a unique number $\beta>\kappa$ such that $f_{1}^{\prime}\left(i \beta, 0^{-}\right)=0$; moreover, we have $f_{1}(i z, 0)>0$ for $z>0, f_{1}^{\prime}\left(i z, 0^{-}\right)<0$ for $z>\beta, f_{1}^{\prime}\left(i z, 0^{-}\right)>0$ for $z \in(0, \beta), T(i z)>0$ for $z>\kappa$, and $T(i z)<0$ for $z \in(0, \kappa)$.

PROOF: Since $U$ has no bound states, it is already known from the spectral theory of ordinary differential equations $[2,17]$ that $f_{1}(0,0)>0$, and that $f_{1}^{\prime}\left(0,0^{+}\right)<0$ if $U$ is 
generic and $f_{1}^{\prime}\left(0,0^{+}\right)=0$ if $U$ is exceptional. Define

$$
\tilde{c}_{0}:=\frac{f_{1}^{\prime}\left(0,0^{+}\right)}{f_{1}(0,0)} .
$$

Thus, $\tilde{c}_{0}=0$ if $U$ is exceptional and $\tilde{c}_{0}<0$ if $U$ is generic. Since $U$ uniquely determines $f_{1}(0,0)$ and $f_{1}^{\prime}\left(0,0^{+}\right)$, the nonpositive constant $\tilde{c}_{0}$ is uniquely determined by $U$ alone. From (1.3) it is seen that if $c_{0}>\tilde{c}_{0}$ then $f_{1}^{\prime}\left(0,0^{-}\right)<0$ and hence $V$ is generic and has no bound states. Consequently, the properties stated in (i) hold. On the other hand, if $c_{0}=\tilde{c}_{0}$, then from (1.3) it follows that $f_{1}^{\prime}\left(0,0^{-}\right)=0$, and hence the corresponding $V$ is exceptional and has no bound states, and consequently the properties stated in (ii) hold. Finally, if $c_{0}<\tilde{c}_{0}$, then from (1.3) it follows that $f_{1}^{\prime}\left(0,0^{-}\right)>0$, which implies that $V$ is generic and possesses one bound state. Consequently, the properties stated in (iii) hold.

Define

$$
M(k):=\frac{f_{1}^{\prime}\left(k, 0^{-}\right)}{i k f_{1}(k, 0)}
$$

From (1.2) it follows that

$$
M(k)=\frac{1-L(k)}{1+L(k)}, \quad L(k)=\frac{1-M(k)}{1+M(k)}
$$

We summarize the relevant properties of $M$ below.

Theorem 2.2 Assume $V$ belongs to class $\mathcal{W}$. Then the following hold:

(i) $M$ is analytic in $\mathbf{C}^{+}$and continuous in $\overline{\mathbf{C}^{+}} \backslash\{0\}$.

(ii) $M$ is continuous at $k=0$ if $V$ is exceptional, and $M$ has a singularity of $O(1 / k)$ at $k=0$ when $V$ is generic.

(iii) If $V$ has no bound states, then $M$ has no zeros in $\overline{\mathbf{C}^{+}}$. If $V$ has one bound state, then $M$ has exactly one zero in $\overline{\mathbf{C}^{+}}$occurring at $k=i \beta$ with $\beta>0$, which corresponds to the only zero of $f_{1}^{\prime}\left(k, 0^{-}\right)$.

(iv) We have $M(k)=\frac{1}{i k} \frac{f_{1}^{\prime}\left(0,0^{-}\right)}{f_{1}(0,0)}+\frac{1}{f_{1}(0,0)^{2}}+o(1)$ as $k \rightarrow 0$ in $\overline{\mathbf{C}^{+}}$. Hence, in the 
exceptional case $M(k)=\frac{1}{f_{1}(0,0)^{2}}+o(1)$ as $k \rightarrow 0$ in $\overline{\mathbf{C}^{+}}$. One can replace o $(1)$ by $O(k)$ if we further have $U \in L_{2}^{1}\left(\mathbf{R}^{+}\right)$.

(v) In the generic case we have $\frac{1}{M(k)}=i k \frac{f_{1}(0,0)}{f_{1}^{\prime}\left(0,0^{-}\right)}+\frac{k^{2}}{f_{1}^{\prime}\left(0,0^{-}\right)^{2}}+o\left(k^{2}\right)$ as $k \rightarrow 0$ in $\overline{\mathbf{C}^{+}}$. One can replace o $\left(k^{2}\right)$ by $O\left(k^{3}\right)$ if we further have $U \in L_{2}^{1}\left(\mathbf{R}^{+}\right)$.

(vi) The real part of $M$ is given by $\operatorname{Re}[M(k)]=\frac{1}{\left|f_{1}(k, 0)\right|^{2}}$ for $k \in \mathbf{R}$.

(vii) The real part of $1 / M$ is given by $\operatorname{Re}\left[\frac{1}{M(k)}\right]=\frac{k^{2}}{\left|f_{1}^{\prime}\left(k, 0^{-}\right)\right|^{2}}$ for $k \in \mathbf{R}$.

(viii) $M(k)=1+\frac{i c_{0}}{k}+o(1 / k)$ as $k \rightarrow \infty$ in $\overline{\mathbf{C}^{+}}$.

PROOF: The well-known properties [1-6] of $f_{1}(k, 0)$ and $f_{1}^{\prime}\left(k, 0^{-}\right)$imply (i)-(iii) and (viii). The asymptotics stated in (iv) and (v) can be found in [18]. The properties in (vi) and (vii) are directly obtained from (2.1) with the help (1.4) and the fact that the Wronskian of $f_{1}(-k, x)$ and $f_{1}(k, x)$ is independent of $x$ and is equal to $2 i k$ [1-6].

Let us define

$$
\begin{gathered}
\ell(k):=\frac{i c_{0}+\left(2 k+i c_{0}\right) L(k)}{2 k-i c_{0}[1+L(k)]}, \quad k \in \overline{\mathbf{C}^{+}}, \\
\tau(k):=\exp \left(\frac{1}{2 \pi i} \int_{-\infty}^{\infty} d t \frac{\log \left(1-|\ell(t)|^{2}\right.}{t-k-i 0^{+}}\right), \quad k \in \overline{\mathbf{C}^{+}}, \\
\rho(k):=-\frac{\ell(-k) \tau(k)}{\tau(-k)}, \quad k \in \mathbf{R}, \\
\hat{\ell}(\alpha):=\frac{1}{2 \pi} \int_{-\infty}^{\infty} d k \ell(k) e^{i k \alpha}, \quad \hat{\rho}(\alpha):=\frac{1}{2 \pi} \int_{-\infty}^{\infty} d k \rho(k) e^{i k \alpha} .
\end{gathered}
$$

The quantities $\tau, \ell$, and $\rho$ correspond to the scattering coefficients for the potential $U$, i.e. the choice $c_{0} \equiv 0$ in $L(k)$ and $T(k)$ leads to $\tau(k)=T(k), \ell(k)=L(k)$, and $\rho(k)=R(k)$, where $R$ is the right reflection coefficient for $V$ and $R(k)=-L(-k) T(k) / T(-k)$.

The following theorem gives the characterization of class $\mathcal{W}$ in terms of the scattering data consisting of $L$ alone. Given $L$ satisfying such characterization conditions, we can uniquely construct the corresponding potential by using any one of the available methods $[1-6]$. 
Theorem 2.3 The left reflection coefficient $L$ corresponds to a unique potential $V$ in $\mathcal{W}$ if and only if the following conditions hold:

(i) $L$ is continuous for $k \in \mathbf{R}$, and $L(-k)=L(k)^{*}$ for $k \in \mathbf{R}$.

(ii) $|L(k)| \leq 1-C k^{2} /\left(1+k^{2}\right)$ on $\mathbf{R}$ for some positive constant $C$.

(iii) $L(0) \in[-1,1)$.

(iv) L either has an analytic extension from $\mathbf{R}$ to $\mathbf{C}^{+}$or it has a meromorphic extension to $\mathbf{C}^{+}$with a single simple pole occurring on the positive imaginary axis, say at $k=i \kappa$ for some $\kappa>0$. In the latter case, the residue of $L$ at $k=i \kappa$ satisfies $\operatorname{Res}[L(i \kappa)]=i c_{\mathrm{r}}^{2}$ for some positive $c_{\mathrm{r}}$.

(v) $L(k)=\frac{c_{0}}{2 i k}+o(1 / k)$ as $k \rightarrow \infty$ in $\overline{\mathbf{C}^{+}}$, where $c_{0}$ is the strength of the delta distribution in $V$ at $x=0$.

(vi) The function $k / \tau(k)$, where $\tau$ is the quantity defined in (2.4), is continuous in $\overline{\mathbf{C}^{+}}$.

(vii) The function $\hat{\ell}(\alpha)$, defined in (2.5) is absolutely continuous on $\mathbf{R}^{-}$and $\hat{\ell}^{\prime}(\alpha) \in$ $L_{1}^{1}(-\infty, 0)$; the function $\hat{\rho}(\alpha)$ defined in (2.5) is absolutely continuous on $\mathbf{R}^{+}$and $\hat{\rho}^{\prime}(\alpha) \in L_{1}^{1}(0,+\infty)$.

PROOF: We modify the characterization conditions on the scattering data $[4,6,19]$ corresponding to a real-valued potential belonging to $L_{1}^{1}(\mathbf{R})$ in order to take into account the presence of the Dirac delta distribution at $x=0$ as well as the vanishing property of the potential on $\mathbf{R}^{-}$. The presence of $c_{0}$ appears in $(\mathrm{v})$. As indicated in Proposition 2.1, the value of $c_{0}$ determines whether $V$ has no bound states or has exactly one bound state, which in turn determines whether the extension of $L$ in $\mathbf{C}^{+}$is analytic or meromorphic. It is known [6,8-13] that (iv) is equivalent to vanishing of $V$ on $\mathbf{R}^{-}$. The slight modification in (vii) is also related to the vanishing of the potential on $\mathbf{R}^{-}$.

Let us note that the constant $c_{\mathrm{r}}$ appearing in Theorem 2.3(iv) corresponds to the bound-state norming constant associated with the right scattering data $[6,8,9]$. 


\section{CONSTRUCTION OF $f_{1}(k, 0)$ AND $f_{1}^{\prime}\left(k, 0^{-}\right)$FROM THEIR AMPLITUDES}

In class $\mathcal{W}$, we can uniquely construct $f_{1}(k, 0)$ for $k \in \overline{\mathbf{C}^{+}}$from its amplitude given for $k \in \mathbf{R}$ by solving the Riemann-Hilbert problem

$$
f_{1}(k, 0) f_{1}(-k, 0)=\left|f_{1}(k, 0)\right|^{2}, \quad k \in \mathbf{R} .
$$

It is known [1-6] that $f_{1}(k, 0)$ is analytic in $\mathbf{C}^{+}$and continuous in $\overline{\mathbf{C}^{+}}, f_{1}(k, 0) \neq 0$ in $\overline{\mathbf{C}^{+}}$ and $f_{1}(k, 0)=1+O(1 / k)$ as $k \rightarrow \infty$ in $\overline{\mathbf{C}^{+}}$. The construction of $f_{1}(k, 0)$ is similar to the construction of the transmission coefficient $T$ from its amplitude $[1,2]$ :

$$
f_{1}(k, 0)=\exp \left(\frac{1}{2 \pi i} \int_{-\infty}^{\infty} d t \frac{\log \left(\left|f_{1}(t, 0)\right|\right)}{t-k-i 0^{+}}\right) .
$$

Now let us consider the construction of $f_{1}^{\prime}\left(k, 0^{-}\right)$for $k \in \overline{\mathbf{C}^{+}}$from its amplitude known for $k \in \mathbf{R}$. Recall that, as indicated in Proposition 2.1, the corresponding potential $V$ has either zero or one bound state. When $V$ has no bound states, we can uniquely construct $f_{1}^{\prime}\left(k, 0^{-}\right)$for $k \in \overline{\mathbf{C}^{+}}$from its amplitude given for $k \in \mathbf{R}$ by solving the Riemann-Hilbert problem

$$
f_{1}^{\prime}\left(k, 0^{-}\right) f_{1}^{\prime}\left(-k, 0^{-}\right)=\left|f_{1}^{\prime}\left(k, 0^{-}\right)\right|^{2}, \quad k \in \mathbf{R} .
$$

It is known [1-6] that $f_{1}^{\prime}\left(k, 0^{-}\right)$is analytic in $\mathbf{C}^{+}$and continuous in $\overline{\mathbf{C}^{+}}$, it has no zeros in $\overline{\mathbf{C}^{+}} \backslash\{0\}$ if $V$ has no bound states, it has exactly one zero if $V$ has a bound state, and $f_{1}^{\prime}\left(k, 0^{-}\right)=i k+O(1)$ as $k \rightarrow \infty$ in $\overline{\mathbf{C}^{+}}$. When there is a bound state, the above Riemann-Hilbert problem leads to a one-parameter family for $f_{1}^{\prime}\left(k, 0^{-}\right)$where the parameter corresponds to the zero of $f_{1}^{\prime}\left(k, 0^{-}\right)$in $\mathbf{C}^{+}$. As indicated in Proposition 2.1, such a zero occurs on the positive imaginary axis in $\mathbf{C}^{+}$.

Thus, the construction of the potential $V$ in $\mathcal{W}$ (or equivalently the left reflection coefficient $L$ ) by using the scattering data $\left|f_{1}(k, 0)\right|$ is equivalent to using the data $f_{1}(k, 0)$. On the other hand, the construction of $V$ in $\mathcal{W}$ from the data $\left|f_{1}^{\prime}\left(k, 0^{-}\right)\right|$is equivalent to the construction using the data $f_{1}^{\prime}\left(k, 0^{-}\right)$only when $V$ has no bound states; when $V$ has 
one bound state, there will be an additional parameter appearing if the data $\left|f_{1}^{\prime}\left(k, 0^{-}\right)\right|$is used instead of the data $f_{1}^{\prime}\left(k, 0^{-}\right)$.

\section{CONSTRUCTION OF $L(k)$ FROM $f_{1}(k, 0)$ OR FROM $\left|f_{1}(k, 0)\right|$}

In the previous section we have shown that the data $f_{1}(k, 0)$ for $k \in \mathbf{R}$ is equivalent to the data $\left|f_{1}(k, 0)\right|$ for $k \in \mathbf{R}$ as far as the construction of a potential in $\mathcal{W}$ is concerned. In this section we show that either data leads to a one-parameter family of potentials, where $c_{0}$, the strength of the Dirac delta distribution in the potential at $x=0$, is the free parameter. In the special case where $c_{0}$ is specified as a part of the data, the construction is unique.

In terms of $M$ given in (2.2), let us define

$$
\Gamma(k):=M(k)+\frac{i}{k} \frac{f_{1}^{\prime}\left(0,0^{-}\right)}{f_{1}(0,0)}-1, \quad k \in \overline{\mathbf{C}^{+}} .
$$

From Theorem 2.2 it follows that $\Gamma$ is analytic in $\mathbf{C}^{+}$, bounded and continuous in $\overline{\mathbf{C}^{+}}$, and

$$
\begin{gathered}
\Gamma(k)=\frac{i}{k}\left[c_{0}-\frac{f_{1}^{\prime}\left(0,0^{-}\right)}{f_{1}(0,0)}\right]+o(1 / k), \quad k \rightarrow \infty \text { in } \overline{\mathbf{C}^{+}}, \\
\operatorname{Re}[\Gamma(k)]=\frac{1}{\left|f_{1}(k, 0)\right|^{2}}-1, \quad k \in \mathbf{R} .
\end{gathered}
$$

Thus, via the Schwarz integral formula, we can construct $\Gamma$ explicitly as

$$
\Gamma(k)=\frac{i}{\pi} \int_{-\infty}^{\infty} \frac{d t}{k+i 0^{+}-t}\left[\frac{1}{\left|f_{1}(t, 0)\right|^{2}}-1\right], \quad k \in \overline{\mathbf{C}^{+}}
$$

Having constructed $\Gamma$, using (4.2), we obtain

$$
\frac{f_{1}^{\prime}\left(0,0^{-}\right)}{f_{1}(0,0)}=-c_{0}-i \lim _{k \rightarrow \infty}[k \Gamma(k)], \quad k \in \overline{\mathbf{C}^{+}}
$$

Then, with the help of (2.3), (4.1), (4.3), and (4.4), we construct $L$ as

$$
L(k)=\frac{-i k \Gamma(k)+c_{0}-i \lim _{k \rightarrow \infty}[k \Gamma(k)]}{2 i k+i k \Gamma(k)-c_{0}+i \lim _{k \rightarrow \infty}[k \Gamma(k)]}, \quad k \in \overline{\mathbf{C}^{+}} .
$$


With the help of (2.2) we also get

$$
f_{\mathrm{l}}^{\prime}\left(k, 0^{-}\right)=i f_{\mathrm{l}}(k, 0)\left[k \Gamma(k)+k+i c_{0}-\lim _{k \rightarrow \infty}[k \Gamma(k)]\right], \quad k \in \overline{\mathbf{C}^{+}}
$$

Since $\Gamma$ is uniquely obtained via (4.3), from (4.5) it is seen that there is a one-parameter family of $L$ corresponding to the data $\left|f_{1}(k, 0)\right|$, where $c_{0}$ acts as the parameter. Consequently, there is a one-parameter family of potentials in $\mathcal{W}$ corresponding to the same scattering data $\left|f_{1}(k, 0)\right|$. On the other hand, if the value of $c_{0}$ is specified in the data, then the construction leads to a unique potential; for example, if the potential is known not to contain a delta distribution at $x=0$, such a potential is uniquely constructed with the help of (4.5) by letting $c_{0}=0$ there.

Note that (1.3) and (4.4) imply

$$
\tilde{c}_{0}=-i \lim _{k \rightarrow \infty}[k \Gamma(k)], \quad \frac{f_{1}^{\prime}\left(0,0^{-}\right)}{f_{1}(0,0)}=\tilde{c}_{0}-c_{0}
$$

where $\tilde{c}_{0}$ is the critical value appearing in Proposition 2.1 that is used to determine whether $V$ has a bound state or not.

Example 4.1 Let us illustrate the above procedure with the scattering data $\left|f_{1}(k, 0)\right|^{2}=$ $\frac{k^{2}+5}{k^{2}+3}$. Solving the Riemann-Hilbert problem in (3.1) one can uniquely determine that $f_{1}(k, 0)=\frac{k+\sqrt{5} i}{k+\sqrt{3} i}$ even though $f_{1}(k, 0)$ is not needed to recover $L$. Evaluating the integral in (4.3) as a contour integral along the lower semicircle of infinite radius centered at the origin of the complex plane, we obtain $\Gamma(k)=\frac{2}{\sqrt{5} i(k+\sqrt{5} i)}$. Then, from (4.5) we get

$$
L(k)=\frac{-i k c_{0}+2+\sqrt{5} c_{0}}{2 k^{2}+i k\left(c_{0}+2 \sqrt{5}\right)-2-\sqrt{5} c_{0}},
$$

which, for each $c_{0} \in \mathbf{R}$ corresponds to a different potential in $\mathcal{W}$. By analyzing the denominator in (4.8), we see that the poles of $L$ occur when

$$
k=\frac{i}{4}\left[-\left(c_{0}+2 \sqrt{5}\right) \pm \sqrt{c_{0}^{2}-4 \sqrt{5} c_{0}+4}\right]
$$


and hence $L$ has a simple pole in $\mathbf{C}^{+}$when $c_{0}<-2 / \sqrt{5}$, which causes the corresponding potential to be generic and to have a bound state. When $c_{0}=-2 / \sqrt{5}$, we obtain $L(k)=$ $\frac{i}{\sqrt{5} k+4 i}$, which corresponds to an exceptional potential without a bound state. When $c_{0}>-2 / \sqrt{5}$, the corresponding potential is generic and has no bound states. Note that with the help of (4.7) we get $\tilde{c}_{0}=-2 / \sqrt{5}$, and hence the criterion for the existence of a bound state is consistent with the results implied by Proposition 2.1. Using any one of the available methods [1-6] to solve the inverse problem, one obtains all these potentials as

$$
V(x)=c_{0} \delta(x)+\theta(x) \frac{24(4+\sqrt{15}) e^{2 \sqrt{3} x}}{\left[(4+\sqrt{15}) e^{2 \sqrt{3} x}-1\right]^{2}},
$$

where $\theta(x)$ denotes the Heaviside function. In this example, from (4.6) we see that

$$
f_{1}^{\prime}\left(k, 0^{-}\right)=i \frac{k^{2}+i k\left(c_{0}+\sqrt{5}\right)-\sqrt{5} c_{0}-2}{k+\sqrt{3} i} .
$$

\section{CONSTRUCTION OF $L(k)$ FROM $f_{1}^{\prime}\left(k, 0^{-}\right)$OR FROM $\left|f_{1}^{\prime}\left(k, 0^{-}\right)\right|$}

In Section 3 we have shown that, in the absence of a bound state, the data $\left|f_{1}^{\prime}\left(k, 0^{-}\right)\right|$is equivalent to the data $f_{1}^{\prime}\left(k, 0^{-}\right)$as far as the construction of a potential in $\mathcal{W}$ is concerned. On the other hand, if there is a bound state, which occurs if $c_{0}$ is sufficiently negative, from $\left|f_{1}^{\prime}\left(k, 0^{-}\right)\right|$one obtains a one-parameter family of $f_{1}^{\prime}\left(k, 0^{-}\right)$whose members differ from each other in the multiplicative rational factor $\frac{k-i \beta}{k+i \beta}$, where the parameter $\beta \in(0,+\infty)$ corresponds to the (only) zero of $f_{1}^{\prime}\left(k, 0^{-}\right)$in $\mathbf{C}^{+}$.

Let us first consider the case where $V$ does not contain a bound state. In this case we show that $\left|f_{1}^{\prime}\left(k, 0^{-}\right)\right|$uniquely determines $L$ and hence also $V$. Define

$$
\Lambda(k):=\frac{1}{M(k)}-1, \quad k \in \overline{\mathbf{C}^{+}}
$$

where $M$ is the quantity in (2.2). From Theorem 2.2 it follows that $\Lambda$ is analytic in $\mathbf{C}^{+}$, bounded and continuous in $\overline{\mathbf{C}^{+}}$, and

$$
\Lambda(k)=-\frac{i c_{0}}{k}+o(1 / k), \quad k \rightarrow \infty \text { in } \overline{\mathbf{C}^{+}}
$$




$$
\operatorname{Re}[\Lambda(k)]=\frac{k^{2}}{\left|f_{1}^{\prime}\left(k, 0^{-}\right)\right|^{2}}-1, \quad k \in \mathbf{R} .
$$

Thus, via the Schwarz integral formula, we can construct $\Lambda$ explicitly as

$$
\Lambda(k)=\frac{i}{\pi} \int_{-\infty}^{\infty} \frac{d t}{k+i 0^{+}-t}\left[\frac{t^{2}}{\left|f_{1}^{\prime}\left(t, 0^{-}\right)\right|^{2}}-1\right], \quad k \in \overline{\mathbf{C}^{+}} .
$$

Having constructed $\Lambda$, using (5.2), we obtain

$$
c_{0}=i \lim _{k \rightarrow \infty}[k \Lambda(k)]
$$

Hence, our data $\left|f_{1}^{\prime}\left(k, 0^{-}\right)\right|$uniquely determines the value of $c_{0}$. Since $V$ does not contain any bound states, we are assured that $c_{0}$ obtained from (5.3) satisfies $c_{0} \geq \tilde{c}_{0}$, where $\tilde{c}_{0}$ is the constant defined in (2.1). Finally, with the help of (2.3), (5.1), and (5.4), we construct $L$ as

$$
L(k)=\frac{\Lambda(k)}{2+\Lambda(k)}, \quad k \in \overline{\mathbf{C}^{+}} .
$$

Note that the above procedure is valid both in the generic and exceptional cases. In the exceptional case, i.e. when $f_{1}^{\prime}\left(0,0^{-}\right)=0$, the quantity $f_{1}^{\prime}\left(k, 0^{-}\right)$vanishes linearly in $k$ as $k \rightarrow 0$ in $\overline{\mathbf{C}^{+}}$, and hence $\Lambda(0)$ is well defined. Note also that, with the help of (2.2), we get

$$
f_{1}(k, 0)=\frac{f_{1}^{\prime}\left(k, 0^{-}\right)}{i k}[\Lambda(k)+1], \quad k \in \overline{\mathbf{C}^{+}} .
$$

Let us illustrate the above procedure with some examples, both in the generic and exceptional cases.

Example 5.1 Let our scattering data be $\left|f_{1}^{\prime}\left(k, 0^{-}\right)\right|^{2}=k^{2}+5$ and assume that $V$ does not have a bound state. Since $\left|f_{1}^{\prime}\left(0,0^{-}\right)\right| \neq 0$, this corresponds to the generic case. Evaluating the integral in (5.4) as a contour integral along the lower semicircle of infinite radius centered at the origin of the complex plane, we obtain $\Lambda(k)=\frac{-\sqrt{5} i}{k+\sqrt{5} i}$. Note that the value of $c_{0}$ is determined uniquely, and from (5.5) we get $\tilde{c}_{0}=\sqrt{5}$. Then, from (5.6) we obtain $L(k)=\frac{-\sqrt{5} i}{2 k+\sqrt{5} i}$, which corresponds to $V(x)=\sqrt{5} \delta(x)$. To construct $V$ uniquely, 
we do not need to construct $f_{1}^{\prime}\left(k, 0^{-}\right)$from our scattering data; nevertheless, as indicated in Section 3, we can uniquely construct it and obtain $f_{1}^{\prime}\left(k, 0^{-}\right)=i(k+\sqrt{5} i)$. Note also that from (5.7) we get $f_{1}(k, 0)=1$.

Example 5.2 Let our scattering data be $\left|f_{1}^{\prime}\left(k, 0^{-}\right)\right|^{2}=\frac{k^{2}\left(5 k^{2}+9\right)}{5\left(k^{2}+3\right)}$ and assume that $V$ does not have a bound state. Even though we do not need to obtain $f_{1}^{\prime}\left(k, 0^{-}\right)$as far as the construction of $L$ is concerned, as pointed out in Section 3, from our scattering data we can uniquely construct $f_{1}^{\prime}\left(k, 0^{-}\right)=\frac{i k(k+3 i / \sqrt{5})}{k+\sqrt{3} i}$. Since $\left|f_{1}^{\prime}\left(0,0^{-}\right)\right|=0$, this corresponds to the exceptional case. Evaluating the integral in (5.4) as a contour integral along the lower semicircle of infinite radius centered at the origin of the complex plane, we obtain $\Lambda(k)=\frac{2 i}{\sqrt{5} k+3 i}$. Note that the value of $c_{0}$ is determined uniquely, and from (5.5) we get $c_{0}=-\frac{2}{\sqrt{5}}$. Then, from (5.6) we obtain $L(k)=\frac{i}{\sqrt{5} k+4 i}$, which corresponds to the unique potential $V$ given by

$$
V(x)=-\frac{2}{\sqrt{5}} \delta(x)+\theta(x) \frac{24(4+\sqrt{15}) e^{2 \sqrt{3} x}}{\left[(4+\sqrt{15}) e^{2 \sqrt{3} x}-1\right]^{2}} .
$$

In this example, from (5.7) we get $f_{1}(k, 0)=\frac{k+\sqrt{5} i}{k+\sqrt{3} i}$.

Next, let us study the recovery of $V$ or equivalently $L$ from the data $\left|f_{1}^{\prime}\left(k, 0^{-}\right)\right|$for $k \in \mathbf{R}$ when $V$ contains one bound state. Given $\left|f_{1}^{\prime}\left(k, 0^{-}\right)\right|$, if we know that $V \in \mathcal{W}$ has a bound state, then we know that $f_{1}^{\prime}\left(k, 0^{-}\right)$has a zero at $k=i \beta$ for some $\beta>0$ even though we do not know the value of $\beta$. If our data is $f_{1}^{\prime}\left(k, 0^{-}\right)$instead of $\left|f_{1}^{\prime}\left(k, 0^{-}\right)\right|$, then we know the value of $\beta$ as a part of our scattering data. In either case, whether $\beta$ is known or not, let us define

$$
\Omega(k):=\frac{k^{2}+\beta^{2}}{k^{2}} \frac{1}{M(k)}-\frac{i \beta^{2}}{k} \frac{f_{1}(0,0)}{f_{1}^{\prime}\left(0,0^{-}\right)}-1, \quad k \in \overline{\mathbf{C}^{+}} .
$$

From Theorem 2.2 it follows that $\Omega$ is analytic in $\mathbf{C}^{+}$, bounded and continuous in $\overline{\mathbf{C}^{+}}$, and we have

$$
\Omega(k)=-\frac{i}{k}\left[c_{0}+\beta^{2} \frac{f_{1}(0,0)}{f_{1}^{\prime}\left(0,0^{-}\right)}\right]+o(1 / k), \quad k \rightarrow \infty \text { in } \overline{\mathbf{C}^{+}},
$$




$$
\operatorname{Re}[\Omega(k)]=\frac{k^{2}+\beta^{2}}{\left|f_{1}^{\prime}\left(k, 0^{-}\right)\right|^{2}}-1, \quad k \in \mathbf{R} .
$$

Thus, via the Schwarz integral formula, we can construct $\Omega$ explicitly as

$$
\Omega(k)=\frac{i}{\pi} \int_{-\infty}^{\infty} \frac{d t}{k+i 0^{+}-t}\left[\frac{t^{2}+\beta^{2}}{\left|f_{1}^{\prime}\left(t, 0^{-}\right)\right|^{2}}-1\right], \quad k \in \overline{\mathbf{C}^{+}} .
$$

Having constructed $\Omega$, using (5.9) we obtain

$$
\beta^{2} \frac{f_{1}(0,0)}{f_{1}^{\prime}\left(0,0^{-}\right)}=-c_{0}+i \lim _{k \rightarrow \infty}[k \Omega(k)]
$$

Then, with the help of $(2.3),(5.8)$, and (5.10), we construct $L$ as

$$
L(k)=\frac{k^{2} \Omega(k)+i k \beta^{2} f_{1}(0,0) / f_{1}^{\prime}\left(0,0^{-}\right)-\beta^{2}}{2 k^{2}+k^{2} \Omega(k)+i k \beta^{2} f_{1}(0,0) / f_{1}^{\prime}\left(0,0^{-}\right)+\beta^{2}}, \quad k \in \overline{\mathbf{C}^{+}} .
$$

From (5.12) we have the following observations. In the presence of a bound state, when one uses the scattering data $\left|f_{1}^{\prime}\left(k, 0^{-}\right)\right|$, we see that there are two free parameters in the constructed $L$, namely $\beta \in(0,+\infty)$ and $f_{1}(0,0)>0$. Thus, the corresponding $V$ also contains these two free parameters. On the other hand, when one uses the scattering data $f_{1}^{\prime}\left(k, 0^{-}\right)$instead, there is only one free parameter, namely $f_{1}(0,0)>0$, in the constructed $L$ and also in $V$. In either case, the value of $c_{0}$ is determined uniquely by using (5.11) once we know both $f_{1}(0,0)$ and $\beta$. In fact, from $(5.9)$ we see that knowledge of any two of the three quantities $c_{0}, \beta$, and $f_{1}(0,0)$ is sufficient for the unique determination of the third. Note also that, with the help of (2.2), (5.9), and (5.11), we get

$$
f_{1}(k, 0)=-\frac{i f_{1}^{\prime}\left(k, 0^{-}\right)}{k^{2}+\beta^{2}}\left[k \Omega(k)+k-i c_{0}-\lim _{k \rightarrow \infty}[k \Omega(k)]\right], \quad k \in \overline{\mathbf{C}^{+}} .
$$

We remark that the procedure given above to recover $f_{1}(k, 0), L(k)$, and $V(x)$ in the presence of one bound state remains valid even when the single bound state of $V$ is not entirely caused by $U$ alone. In other words, there are three ways for $V$ to have one bound state [16]. The first case is that $U$ has two bound states but the large $c_{0}$ value causes $V$ to have one bound state. The second case is when $U$ has one bound state and the value of $c_{0}$ 
is moderate enough so that also $V$ has one bound state. The third case is when $V \in \mathcal{W}$, i.e. when $U$ has no bound states but the large negative value of $c_{0}$ causes $V$ to have one bound state. Our procedure of recovery of $f_{1}(k, 0), L(k)$, and $V(x)$ is valid in all these three cases.

Let us illustrate our recovery procedure when $V$ has one bound state with some concrete examples.

Example 5.3 Let our scattering data be given by $f_{1}^{\prime}\left(k, 0^{-}\right)=\frac{i(k-i)(k+\sqrt{5} i)}{k+i}$. Note that $f_{1}^{\prime}\left(k, 0^{-}\right)$has a zero in $\mathbf{C}^{+}$, and hence the corresponding $V$ contains exactly one bound state. We also see that $f_{1}^{\prime}\left(0,0^{-}\right)=\sqrt{5}$. In our procedure to construct $L$ and $V$, we see that we need to use $\beta=1$. Evaluating (5.10) with the help of a contour integral, we obtain $\Omega(k)=\frac{-4 i}{\sqrt{5}(k+\sqrt{5} i)}$. Then, from $(5.12)$ we obtain the one-parameter family of reflection coefficients

$$
L(k)=\frac{-i k^{2}\left[4-f_{1}(0,0)\right]-\sqrt{5} k\left[1+f_{1}(0,0)\right]-5 i}{2 \sqrt{5} k^{3}+i k^{2}\left[6+f_{1}(0,0)\right]+\sqrt{5} k\left[1-f_{1}(0,0)\right]+5 i},
$$

where $f_{1}(0,0)$ is the parameter. Note that from (5.11) we obtain $c_{0}=\frac{4-f_{1}(0,0)}{\sqrt{5}}$. The one-parameter family of potentials corresponding to (5.14) is given by

$$
V(x)=\frac{4-f_{1}(0,0)}{\sqrt{5}} \delta(x)+\theta(x) U(x),
$$

where $U(x)$ contains the parameter $f_{1}(0,0)$ and can be evaluated explicitly, but we will not display it here because that expression is very lengthy. In this example, from (5.13) we get

$$
f_{1}(k, 0)=\frac{k^{2}+i k\left(\sqrt{5}-c_{0}\right)+\sqrt{5} c_{0}-4}{(k+i)^{2}} .
$$

Thus, we have $f_{1}(0,0)=4-\sqrt{5} c_{0}$, confirming what can also be obtained from (5.15). With the help of (1.3) we get

$$
f_{1}^{\prime}\left(0,0^{+}\right)=f_{1}^{\prime}\left(0,0^{-}\right)+c_{0} f_{1}(0,0)=\sqrt{5}+c_{0}\left(4-\sqrt{5} c_{0}\right)=\sqrt{5}\left(c_{0}-\sqrt{5}\right)\left(c_{0}+1 / \sqrt{5}\right) .
$$

Thus, there are several cases depending on the sign of $f_{1}^{\prime}\left(0,0^{+}\right)$. If $c_{0}<-1 / \sqrt{5}$ or equivalently $f_{1}(0,0)>5$, then $U$ is generic and has no bound states. If $c_{0}=-1 / \sqrt{5}$ then $U$ 
is exceptional and has no bound states. If $c_{0} \in(-1 / \sqrt{5}, \sqrt{5})$ then $U$ is generic and has one bound state. If $c_{0}=\sqrt{5}$ then $U$ is exceptional and has one bound state. Finally, if $c_{0}>\sqrt{5}$ then $U$ is generic and has two bound states.

Example 5.4 Let our scattering data be given by $\left|f_{1}^{\prime}\left(k, 0^{-}\right)\right|^{2}=k^{2}+5$ and let us assume that it is known that $V$ contains a bound state. In this case, corresponding to our data, we can construct the one-parameter family $f_{1}^{\prime}\left(k, 0^{-}\right)=\frac{i(k-i \beta)(k+\sqrt{5} i)}{k+i \beta}$, where $\beta>0$ is the parameter. Thus, we know that $f_{1}^{\prime}\left(0,0^{-}\right)=\sqrt{5}$. From (5.10) we obtain

$$
\Omega(k)=\frac{i\left(\beta^{2}-5\right)}{\sqrt{5}(k+\sqrt{5} i)},
$$

and hence, via (5.12), we get

$$
L(k)=\frac{i k^{2}\left[\beta^{2}-5+\beta^{2} f_{1}(0,0)\right]-\sqrt{5} \beta^{2} k\left[1+f_{1}(0,0)\right]-5 i \beta^{2}}{2 \sqrt{5} k^{3}+i k^{2}\left[5+\beta^{2}+\beta^{2} f_{1}(0,0)\right]+\sqrt{5} \beta^{2} k\left[1-f_{1}(0,0)\right]+5 i \beta^{2}},
$$

where $\beta$ and $f_{1}(0,0)$ are the two arbitrary positive parameters. Note that (5.11) gives us

$$
c_{0}=\frac{5-\beta^{2}-\beta^{2} f_{1}(0,0)}{\sqrt{5}} .
$$

The two-parameter family of potentials corresponding to (5.16) is given by

$$
V(x)=\frac{5-\beta^{2}-\beta^{2} f_{1}(0,0)}{\sqrt{5}} \delta(x)+\theta(x) U(x),
$$

where $U(x)$ contains the two parameters $\beta$ and $f_{1}(0,0)$ and can be evaluated explicitly, but we will not display it here because that expression is very lengthy. Note also that from (5.13) we get

$$
f_{1}(k, 0)=\frac{k^{2}+i k\left(\sqrt{5}-c_{0}\right)+\sqrt{5} c_{0}+\beta^{2}-5}{(k+i \beta)^{2}} .
$$

As in Example 5.3, by analyzing the sign of $f_{1}^{\prime}\left(0,0^{+}\right)$, we can conclude that $U$ has two bound states if $c_{0}>\sqrt{5}$, has one bound state if $c_{0} \in\left(-\beta^{2} / \sqrt{5}, \sqrt{5}\right]$, and has no bound states if $c_{0} \leq-\beta^{2} / \sqrt{5}$.

Acknowledgments. The author has benefited from discussions with John Sylvester, Roy Pike, and Ricardo Weder. The research leading to this article was supported in part by 
the National Science Foundation under grant DMS-0204437 and the Department of Energy under grant DE-FG02-01ER45951.

\section{REFERENCES}

[1] L. D. Faddeev, Properties of the S-matrix of the one-dimensional Schrödinger equation, Amer. Math. Soc. Transl. (Ser. 2) 65, 139-166 (1967).

[2] P. Deift and E. Trubowitz, Inverse scattering on the line, Comm. Pure Appl. Math. 32, 121-251 (1979).

[3] R. G. Newton, The Marchenko and Gel'fand-Levitan methods in the inverse scattering problem in one and three dimensions, In: J. B. Bednar et al. (eds), Conference on inverse scattering: theory and application, SIAM, Philadelphia, 1983, pp. 1-74.

[4] V. A. Marchenko, Sturm-Liouville operators and applications, Birkhäuser, Basel, 1986.

[5] K. Chadan and P. C. Sabatier, Inverse problems in quantum scattering theory, 2nd ed., Springer, New York, 1989.

[6] T. Aktosun and M. Klaus, Chapter 2.2.4, Inverse theory: problem on the line, In: E. R. Pike and P. C. Sabatier (eds.), Scattering, Academic Press, London, 2001, pp. $770-785$.

[7] L. Ahlfors, Complex analysis, 2nd ed., McGraw-Hill, New York, 1966.

[8] N. N. Novikova and V. M. Markushevich, Uniqueness of the solution of the onedimensional problem of scattering for potentials located on the positive semiaxis, Comput. Seismology 18, 164-172 (1987).

[9] T. Aktosun, M. Klaus, and C. van der Mee, On the Riemann-Hilbert problem for the one-dimensional Schrödinger equation, J. Math. Phys. 34, 2651-2690 (1993).

[10] T. Aktosun, Bound states and inverse scattering for the Schrödinger quation in one dimension, J. Math. Phys. 35, 6231-6236 (1994). 
[11] B. Grébert and R. Weder, Reconstruction of a potential on the line that is a priori known on the half line, SIAM J. Appl. Math. 55, 242-254 (1995).

[12] T. Aktosun, Inverse Schrödinger scattering on the line with partial knowledge of the potential, SIAM J. Appl. Math. 56, 219-231 (1996).

[13] F. Gesztesy and B. Simon, Inverse spectral analysis with partial information on the potential. I. The case of an a.c. component in the spectrum, Helv. Phys. Acta 70, 66-71 (1997).

[14] K. Chadan, Chapter 2.2.1, Radial inverse scattering problems, In: E. R. Pike and P. C. Sabatier (eds.), Scattering, Academic Press, London, 2001, pp. 726-741.

[15] L. D. Faddeev, The inverse problem in the quantum theory of scattering, J. Math. Phys. 4, 72-104 (1963).

[16] T. Aktosun, M. Klaus, and C. van der Mee, On the number of bound states for the one-dimensional Schrödinger equation, J. Math. Phys. 39, 4249-4256 (1998).

[17] E. A. Coddington and N. Levinson, Theory of ordinary differential equations, McGrawHill, New York, 1955.

[18] T. Aktosun and M. Klaus, Small-energy asymptotics for the Schrödinger equation on the line, Inverse Problems 17, 619-632 (2001).

[19] A. Melin, Operator methods for inverse scattering on the real line, Comm. Partial Differential Equations 10, 677-766 (1985). 